\title{
Endozoocoria por el zorro gris pampeano, Lycalopex gymnocercus, (Carnivora: Canidae) y germinación del tala, Celtis ehrenbergiana (Rosales: Cannabaceae)
}

\author{
Candela Duarte ${ }^{1}$ (iD) \& Claudia M. Dellafiore ${ }^{1}$ (iD) \\ 1. Universidad Nacional de Río Cuarto, ruta 36 km 601, Río Cuarto, Córdoba, Argentina; cdbaschini@gmail.com; \\ cdellafiore@exa.unrc.edu.ar
}

Recibido 02-IX-2019 • Corregido24-X-2019 • Aceptado 19-I-2020

DOI: https://doi.org/10.22458/urj.v12i1.2615

\begin{abstract}
Endozoochory by pampas fox, Lycalopex gymnocercus, (Carnivora: Canidae) and germination of tala, Celtis ehrenbergiana (Rosales: Cannabaceae)". Introduction: It has been observed that foxes (Lycalopex gymnocercus) consume fruits of "tala" (Celtis ehrenbergiana), a native species of Córdoba, Argentina. This does not necessarily mean that there is a mutualistic relationship, because the seeds can suffer damage in the digestive tract. Objetive: a) To know if the seeds suffer physical damage in the digestive tract, b) To know if this passage affects the viability, germination power, and beginning and germination rate, and c) To know if the viability and germination power change after the seeds have long remained inside the feces. Methods: We compared the variables under study between fruit seeds, seeds in fresh feces, and seeds in six year old feces. Results: $100 \%$ of seeds from feces were healthy; but germination start was faster from seeds extracted from feces. Conclusion: Foxes can disperse $C$. ehrenbergiana seeds and accelerate their germination, which retain their viability even after long periods in the feces.
\end{abstract}

Keywords: Seed dispersal, viability, germination, feces, Argentina.
RESUMEN. Introducción: Se ha observado que los zorros (Lycalopex gymnocercus) consumen frutos de "tala" (Celtis ehrenbergiana), una especie nativa de Córdoba, Argentina. Esto no implica que exista una relación mutualista, ya que las semillas pueden sufrir daños al pasar a través del tracto digestivo. Objetivos: a) Conocer si las semillas sufren daño físico al pasar a través del tracto digestivo, b) Conocer si dicho paso afecta la viabilidad, el poder germinativo, el inicio y tasa de germinación, y c) Conocer si dicho efecto cambia luego de largos períodos de permanencia de las semillas en el interior de las fecas. Métodos: Comparamos las variables en estudio entre las semillas de frutos y las obtenidas de fecas frescas y de fecas de seis años de antigüedad. Resultados: El 100\% de las semillas estaban sanas, pero el inicio de germinación fue más rápido en semillas provenientes de las fecas. Conclusión: Los zorros pueden dispersar semillas de $C$. ehrenbergiana y acelerar su germinación, que conservan su viabilidad aún luego de largos períodos de tiempo en las fecas.

Palabras clave: Dispersión de semillas, viabilidad, germinación, fecas, Argentina. 
La endozoocoria es el proceso de dispersión de semillas mediante animales frugívoros y constituye la relación mutualista más frecuente entre los vertebrados y las plantas. En los bosques tropicales más del 75\% de las especies leñosas dependen de los animales para la dispersión de sus semillas (Janzen \& Vázquez-Yanes, 1991) y, en algunos casos, las semillas necesitan pasar por el tracto digestivo de los mismos para poder germinar (Bascompte \& Jordano, 2008).

Entre los vertebrados, el zorro gris pampeano (Lycalopex gymnocercus) posee una dieta omnívora con un elevado consumo de frutos los cuales pueden llegar a constituir más del $50 \%$ de la misma (Nuñez \& Bozzolo, 2006; Varela \& Bucher, 2008). En los bosques serranos de la provincia de Córdoba se ha observado que los zorros consumen tanto frutos de especies exóticas como de especies nativas entre los que se encuentran los frutos de Celtis ehrenbergiana (tala) (Dellafiore, obs pers). El hecho de que los zorros consuman dichos frutos no implica necesariamente que exista una relación mutualista entre ambas especies ya que el paso a través del tracto digestivo de los animales puede afectar negativamente a las semillas, ya sea en forma mecánica y/o afectando su viabilidad (entendiendo por viabilidad la capacidad de la semilla para germinar en condiciones favorables).

De acuerdo con las condiciones ambientales las fecas de los carnívoros pueden permanecer sin degradarse sobre el sustrato durante largos períodos de tiempo que van desde días a años (Chin, 2002; Noss \& Maffei, 2013; Lonsinger, Gese, Knight, Johnson, \& Waits, 2016). En el caso del zorro se ha observado que las fecas pueden secarse y endurecerse persistiendo durante largos períodos de tiempo sin descomponerse (Dellafiore, obs pers) por lo que la viabilidad y poder germinativo de las semillas de Celtis ehrenbergiana podrían verse modificados de acuerdo al tiempo de permanencia en el interior de las mismas.

De acuerdo con lo expuesto anteriormente, este estudio tuvo por objetivos: a) conocer si las semillas de $C$. ehrenbergiana sufren daño físico al pasar a través del tracto digestivo de los zorros, b) conocer si dicho paso afecta la viabilidad, el poder germinativo, el inicio y la tasa de germinación y c) conocer si dicho efecto cambia luego de largos períodos de permanencia de las semillas en el interior de las fecas.

\section{MATERIALES Y MÉTODOS}

Celtis ehrenbergiana (tala) pertenece a la familia de las Cannabaceae, es nativa de América y se distribuye desde América del Norte hasta el centro-sur de América del Sur (hasta el sur de la provincia de Buenos Aires) (Biloni, 1990). Esta especie florece en los meses de octubre-noviembre y fructifica de diciembre a marzo (Romanczuk \& Del Pero De Martínez, 1978). El fruto es una drupa poco carnosa, color amarillo-anaranjado en la madurez y en su interior posee una sola semilla dura, rugosa, de forma esférica de unos 4 o $5 \mathrm{~mm}$ (obs pers). Esta especie es una importante fuente de alimento tanto para las aves como para los mamíferos del bosque y matorral serrano (Dellafiore, 2016; Dellafiore \& Rosa, 2017; Dellafiore obs.pers.).

Para estudiar el efecto del paso de las semillas a través del tracto digestivo del zorro se comparó la germinación entre las semillas recolectadas directamente de los frutos y las obtenidas de las fecas del zorro. La recolección de frutos y de fecas se llevó a cabo a orillas del Río Chocancharava en la zona noreste de la ciudad de Río Cuarto, provincia de Córdoba, Argentina. El área de estudio posee una superficie de 12 ha y la vegetación presente corresponde a la región fitogeográfica pampeana - distrito occidental (Cabrera, 1976). La temperatura media anual es de $18^{\circ} \mathrm{C}$ con una precipitación media anual de $800 \mathrm{~mm}$. En dicha área de estudio se recolectaron 5000 frutos de $C$. ehrenbergiana de 10 plantas diferentes. A dichos frutos se les extrajeron las semillas y se seleccionaron al azar diez muestras de 30 semillas cada una. Cada muestra fue sembrada en placas germinadoras individuales (ver detalle debajo). Por otro lado, durante enero de 2018 se 
colocaron dos transectas de $1000 \mathrm{~m}$ de largo por $50 \mathrm{~m}$ de ancho las cuales fueron revisadas y las fecas de zorro fueron eliminadas para asegurase de que las fecas recolectadas durante los muestreos no tuvieran más de un mes de antigüedad. Durante febrero y marzo del mismo año se revisaron nuevamente dichas transectas y se recolectaron 20 y 14 fecas de zorro respectivamente. Dichas fecas fueron llevadas al laboratorio, se desarmaron suavemente y las semillas de $C$. ehrenbergiana fueron separadas y observadas bajo lupa estereoscópica para registrar posibles daños físicos (por ejemplo, semillas partidas, tegumento roto, exposición del embrión, deshidratación, etc.). Se obtuvieron 545 semillas, todas estaban sanas, y se tomaron al azar 10 muestras de 30 semillas cada una las cuales fueron sembradas para su germinación (ver detalle debajo). El tiempo transcurrido entre la recolección de las muestras y la siembra fue de siete días. Durante este período las muestras fueron conservadas en sobres de papel a temperatura ambiente.

Para evaluar si el efecto del paso de las semillas a través del tracto digestivo del zorro puede verse modificado en función del tiempo que permanecen en las fecas, se analizaron 20 muestras recolectadas durante marzo de 2012. Dichas muestras fueron conservadas en sobres de papel a temperatura ambiente y en marzo de 2018 se analizaron siguiendo el mismo procedimiento al mencionado previamente. Se obtuvieron 210 semillas de $C$. ehrenbergiana, todas estaban sanas, y fueron puestas a germinar en siete placas de 30 semillas cada una.

En todos los casos las semillas fueron sembradas en placas de Petri con papel secante, se colocaron en germinador a temperatura constante de $25^{\circ} \mathrm{C}$ y se regaron diariamente. Dichas placas se revisaron cada dos días en búsqueda de evidencias de germinación durante 234 días. El criterio de germinación fue la emergencia de la radícula.

El inicio de la germinación (GS) y la tasa de germinación (GR) fueron evaluadas siguiendo el método de Izhaki y Ne'eman (1997). De acuerdo con estos autores el inicio de la germinación se define como el intervalo (días) entre la siembra y la germinación y se calculó como:

$$
\mathrm{GS}=1 / 6 * \mathrm{P}
$$

donde, P es el porcentaje final de germinación.

La tasa de germinación se calculó como:

$$
\mathrm{GR}=(5 / 6 * \mathrm{P}) /(\mathrm{T} 2-\mathrm{T} 1)
$$

donde, $\mathrm{T} 1$ es el intervalo de tiempo (días) entre la siembra y la germinación de $1 / 6 * \mathrm{P}$ de las semillas, y $\mathrm{T} 2$ es el intervalo de tiempo (días) entre la siembra y la emergencia de $5 / 6$ * P de plántulas.

El poder germinativo (PG) representa el porcentaje final de semillas que germinan $(\mathrm{Sg})$ con relación al número total de semillas sembradas (Ss):

$$
P G=(S g / S s) \times 100 \%
$$

Se calculó el porcentaje acumulado de germinación entre las semillas de los frutos y las fecas a lo largo del tiempo y la prueba de la $t$ para muestras independientes fue empleada para detectar diferencias significativas entre los tratamientos.

Por último, se aplicó la prueba de tetrazolio a las semillas que no germinaron al finalizar el experimento para conocer su viabilidad (Cottrell, 1947; MacKay, 1972). Para ello las semillas fueron cortadas por la mitad y se sumergieron en una solución acuosa al $1 \%$ de cloruro de 2, 3, 5 - trifenil - tetrazolio, $\mathrm{pH} 7$, en la oscuridad durante $72 \mathrm{~h}$ a una temperatura constante de $25^{\circ} \mathrm{C}$. Finalmente, el 
embrión se observó bajo lupa estereoscópica; los teñidos de rojo eran viables y los no coloreados eran inviables (Bradbeer, 1988).

Ética, conflicto de intereses y declaración de financiamiento Los autores declaran haber cumplido con todos los requisitos éticos y legales pertinentes, tanto durante el estudio como en el manuscrito; que no hay conflictos de interés de ningún tipo, y que todas las fuentes financieras se detallan plena y claramente en la sección de agradecimientos. Asimismo, están de acuerdo con la versión editada final del documento. El respectivo documento legal firmado se encuentra en los archivos de la revista.

\section{RESULTADOS}

El 100\% de las semillas recolectadas de las fecas de zorro de 2012 y 2018 estaban sanas; es decir no presentaron evidencias de daño físico luego de pasar por el tracto digestivo de los animales.

Se observaron diferencias significativas en los porcentajes de germinación acumulada entre las semillas de los frutos y las obtenidas de las fecas de 2018 ( $t=2,42, p=0,016)$ y 2012 ( $t=2,57$, $p=0,01)$. No se encontraron diferencias significativas entre las semillas de las fecas de 2018 y 2012 (Fig. 1).

El inicio de germinación (GS) fue significativamente más bajo para las semillas provenientes de las fecas que para las semillas obtenidas de los frutos (semilla frutos vs semilla fecas de 2018: $t=1,66, p=0,03$; semilla frutos vs semilla fecas de 2012: $t=4,56, p=0,06$ ) (Fig. 1). Las semillas de los frutos comenzaron a germinar a los $77 \pm 11$ días y continuaron germinando hasta el día 172 . Las semillas provenientes de las fecas recolectadas durante 2018 comenzaron a germinar a los $46 \pm 11$ días y continuaron germinando hasta el día 176 mientras que las semillas provenientes de las fecas de 2012 comenzaron a germinar a los 44_3 días y la germinación ocurrió hasta el día 129 (Fig. 1).
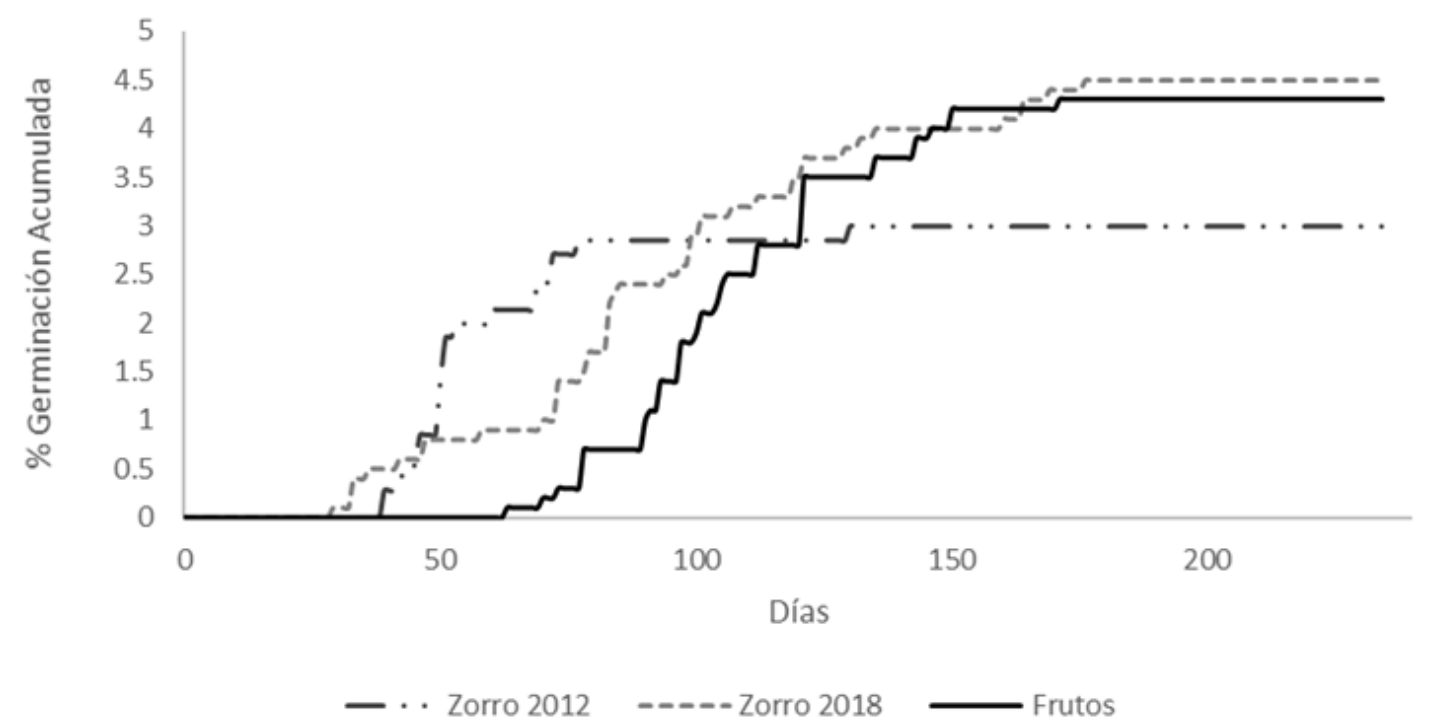

Fig. 1. Porcentaje de germinación acumulado (\%) a través del tiempo (días) de las semillas de Celtis ehrenbergiana recolectadas de los frutos (línea negra) y de las fecas de zorro de 2018 (línea gris de guiones y de las fecas de zorro de 2012 (línea negra con guion y puntos).

No se observaron diferencias significativas entre el poder germinativo (PG) de las semillas de los diferentes tratamientos el cual oscilo entre el $14,3 \pm 7,6 \%$ para las semillas de los frutos, $15 \pm 5,3 \%$ para las semillas de las fecas de 2018 y 10 $\pm 4,2 \%$ para las semillas de las fecas de 2012 (10\%). Tampoco se encontraron diferencias significativas en la tasa de germinación (GR) entre tratamientos 
las cuales fueron de $0,20 \pm 0,43$ para las semillas de los frutos, $0,17 \pm 0,19$ para las semillas de fecas de zorros de 2018, 0,309 $\pm 0,18$ para las semillas de las fecas de 2012 .

La prueba de viabilidad de tetrazolio mostró que el $98 \%, 93 \%$ y $99 \%$ de las semillas de los frutos, de las fecas de 2018, y de las fecas de 2012 respectivamente eran inviables al cabo de 234 días.

\section{DISCUSIÓN}

La dispersión legítima por animales se produce cuando las semillas depositadas en las fecas se encuentran sanas (Fleming \& Sosa, 1994). De acuerdo con los resultados obtenidos, $C$. ehrenbergiana está siendo legítimamente dispersada por el zorro ya que las semillas no sufren daño físico al pasar a través del tracto digestivo de los animales.

El inicio de germinación (GS) fue significativamente menor para las semillas provenientes de las fecas que para las obtenidas de los frutos. Esto podría deberse a que los jugos gástricos contribuyen a degradar la cubierta seminal mejorando la absorción de agua y/o oxígeno y/o la recepción de señales para la germinación (Traveset, 1998). Este inicio de germinación más temprano puede resultar ser una ventaja para $C$. ehrenbergiana ya que una diferencia de unos pocos días puede suponer una mejoría para las plantas en cuanto a una disminución en el riesgo de predación, una mejora de la tasa de crecimiento, una reducción en la probabilidad de muerte y/o reducción de competidores, como ha sido observado para diversas especies vegetales (Symonides, 1977; Garwood, 1986, Travest, 1998).

El ritmo de germinación (GR) y el porcentaje final de germinación (PG) no difirió entre las semillas de los frutos y de las fecas aun cuando las semillas habían permanecido en las fecas durante seis años. Resultados similares han sido observados para Cyanocompsa brissonii aunque difieren de los observado para Turdus serranus quien aumenta el poder germinativo de $C$. ehrenbergiana (Dellafiore \& Rosa, 2017).

El hecho de que las semillas permanezcan viables en el interior de las fecas por largos períodos de tiempo puede ser beneficio para $C$. ehrenbergiana ya que podrían permanecer en su interior hasta encontrar las condiciones favorables para la germinación y a su vez estarían menos expuestas a los riesgos de predación e incluso a condiciones climáticas adversas como ha sido observado por otros autores (Janzen \& Vásquez-Yanes, 1991; Herrera, 2002). Mayores estudios serían necesarios para corroborar esta hipótesis.

De acuerdo con lo expuesto anteriormente observamos que el principal beneficio que obtendría $C$. ehrenbergiana a la hora de ser consumidas por los zorros es poder ser dispersadas viables a áreas alejadas de la planta madre pudiendo alcanzar nuevas áreas abiertas a la colonización. Por otro lado, las semillas se beneficiarían germinando con anterioridad a las semillas de los frutos y este efecto no se perdería, aunque las semillas permanezcan largos períodos de tiempo en el interior de las fecas.

\section{AGRADECIMIENTOS}

Agradecemos a la Secretaría de Ciencia y Técnica de la Universidad Nacional de Río Cuarto por al apoyo financiero. 


\section{REFERENCIAS}

Bascompte, J., \& Jordano P. (2008). Redes mutualistas de especies. Investigación y Ciencia, 384, 50-59.

Biloni, J. S. (1990). Árboles autóctonos Argentinos. Buenos Aires, Argentina: Tipográfica Editora Argentina.

Bradbeer, J. W. (1988). Seed dormancy and germination. USA: Chapman and Hall. DOI: 10.1007/978-1-4684-7747-4

Cabrera, A. L. (1976). Regiones fitogeográficas Argentinas (2 ed.). Enciclopedia Argentina Agrícola y Jardinería. Buenos Aires, Argentina: ACME.

Chin, K. (2002). Analyses of coprolites produced by carnivorous vertebrates. Paleontological Society Papers, 8, 43-49. DOI: $10.1017 / \mathrm{S} 1089332600001042$

Cottrell, H. J. (1947). Tetrazolium salt as a seed germination indicator. Nature, 159, 1-748. DOI: 10.1038/159748a0

Dellafiore, C. M. (2016). Dispersión legítima de semillas por aves en el bosque y matorral serrano de la provincia de Córdoba. European Scientific Journal, 12(18), 56-64. DOI: 10.19044/esj.2016.v12n18p56

Dellafiore, C. M. \& Rosa M. J. (2017). Efecto del zorzal (Turdus serranus) y Reina mora (Cyanocompsa brissonii) sobre la germinación del tala (Celtis ehrebergiana). Revista Gestión Ambiental, 33,5-11

Fleming, T. H., \& Sosa, V. J. (1994). Effects of nectarivorous and frugivorous mammals on reproductive success of plants. Journal of Mammalogy, 75(4), 845-851. DOI: 10.2307/1382466

Garwood, N. C. (1986). Constraints on the timing of seed germination in a tropical forest. In A. Estrada \& T.H. Fleming (Eds.), Frugivores and Seed Dispersal (pp. 347-355) Dordrecht, Netherlands: Springer. DOI: 10.1007/978-94-0094812-9_30

Herrera, C. M. (2002). Seed dispersal by vertebrates. In C. M. Herrera \& O. Pellmyr (Eds.), Plant - Animal Interactions. An Evolutionay Approach (pp. 185-208). Boston, USA: Blackwell Science.

Izhaki, I., \& Ne'eman, G. (1997). Hares (Lepus spp.) as seed dispersers of Retama raetam (Fabaceae) in sandy landscape. Journal of Arid Environments, 37, 343-354. DOI: 10.1006/jare.1997.0273

Jansen, D.H., \& Vázquez-Yanes C. (1991). Aspects of tropical seed ecology of relevance to management of tropical forested wildlands. In A. Gómez-Pompa, T. C. Whitmore \& M. Hadley (Eds.), Rain Forest Regeneration and Management (Vol. 6, pp. 137-157). Paris, France: Man and Biosphere Program, UNESCO.

Lonsinger, R. C., Gese, E. M., Knight, R. N., Johnson, T. R., \& Waits L. P. (2016). Quantifying and correcting for scat removal in noninvasive carnivore scat surveys. Wildlife Biology, 22, 45-54. DOI: 10.2981/wlb.00179

Mackay, D. B. (1972). The measurement of viability. In E. H. Roberts (Ed.), Viability of seeds (pp. 172-208). Netherlands: Springer. DOI: 10.1007/978-94-009-5685-8_7

Noss, A. \& Maffei, L. (2013). Estudio de heces: reconocimiento, registro y almacenaje. Nueva York, USA: Jaguar Conservation Program of Wildlife Conservation Society in Nicaragua.

Nuñez, M. B., \& Bozzolo, L. (2006). Descripción de la dieta del zorro gris, Pseudalopex griseus (Canidae) (Gray, 1842), en el Parque Nacional Sierra de las Quijadas, Argentina. Gayana Zooogy, 70(2), 163-167. DOI: 10.4067/S071765382006000200002

Romanczuk, M. C., \& Del Pero De Martínez, M. A. (1978). Las especies del género Celtis (Ulmaceae) de la Argentina. Darwiniana, 21, 541-577.

Symonides, E. (1977). Mortality of seedlings in natural psammophyte populations. Ekologia Polska, 25, 635-651. 
Traveset, A. (1998). Effect of seed passage through vertebrate frugivores' guts on germination: a review. Perspectives in Plant Ecology. Evolution and Systematics, 2,151-190. DOI: 10.1078/1433-8319-00057

Varela, O., \& Bucher, E. H. (2008). Passage time, viability, and germination of seeds ingested by foxes. Journal of Arid Environments, 67, 566-578. DOI: 10.1016/j.jaridenv.2006.03.013 